\title{
Study Over Acute Appendicitis with Special Reference to the Role of WBC Count, C-Reactive Protein (CRP), USG and Alvarado Score in Making Diagnosis
}

\author{
Authors \\ Dr Pradeep Jaiswal ${ }^{1}$, Dr Anurag Kumar ${ }^{2}$, Dr P. K. Jha ${ }^{3}$, Dr Rajnish Chandran ${ }^{4}$ \\ Dr Digbijay Kumar Singh ${ }^{5}$ \\ ${ }^{1,4}$ Asst. Prof., IGIMS, Patna \\ ${ }^{2,5}$ Sen. Resident, IGIMS, Patna \\ ${ }^{3}$ Additional Prof., IGIMS, Patna
}

\section{Abstract}

Study was done on 140 patient, in IGIMS, Patna for the period of February 2016 to July 2017. In this study attempts was made to undergo a thorough evaluation of all cases of acute appendicitis on the basis of clinical symptoms, physical examinations, some readily available, relatively inexpensive investigations (White Blood Count, C-Reactive Protein, Ultrasonography), and the Alvarado score.

\section{Introduction}

The importance of appendix in surgery results from its propensity for inflammation, which leads to the clinical syndrome known as acute appendicitis. Acute appendicitis is the most common cause of an "acute abdomen" in young adults, and appendectomy is the most frequently performed urgent abdominal operation ${ }^{1}$ with a lifetime prevalence of approximately 1 in 7 . $^{2}$

\section{Aims and Objectives}

1. To study the various modes of presentation of acute appendicitis.

2. To study the different clinical signs elicited in acute appendicitis.

3. To study the role of various investigative procedures with special reference to white blood corpuscle (WBC) count, c-reactive protein (CRP) and ultrasonography (USG) in the diagnosis of acute appendicitis.

4. To evaluate the role of Alvarado scoring system in the diagnosis of acute appendicitis.

5. To find out a way to increase correct diagnosis of acute appendicitis, thereby decreasing negative appendectomy or unnecessary delay in operation.

\section{Clinical Evaluation}

Incidence

Addiss and associates ${ }^{49}$ estimated the incidence of acute appendicitis in the United States population to be 11 cases per 10,000 populations annually.

\section{Symptoms}

\section{Pain abdomen}

Classically, the pain migrates to the right lower quadrant as transmural inflammation of the 
appendix leads to inflammation of the peritoneal lining of the right lower abdomen. This usually occurs within 12-24 hours.

Nausea and vomiting: The pain is typically followed by nausea. Vomiting may or may not be present

\section{Anorexia:}

Fever: Patients may report low-grade fever up to $101^{\circ} \mathrm{F}\left(38.3^{\circ} \mathrm{C}\right)$.

Constipation: Constipation is the usual feature.

Diarrhea: May occur in some cases, particularly in pelvic and postileal varieties Increased frequency of micturation and dysurea: May occurs specially in pelvic variety.

\section{Signs}

General appearance: Patients look mildly ill. They often lie still to avoid the peritoneal irritation caused by movement.

- Pulse: Normal or slightly elevated.

- Temperature: May have slightly elevated temperature.

- Tenderness: Maximal tenderness is typically in the right lower quadrant, at or near McBurney's point,.

- Muscle guarding: This tenderness is often associated with localized muscle rigidity in right iliac fossa

- Rebound tenderness: Rebound, shake, or tap tenderness usually present over right iliac fossa.

- Pointing sign: The patient is asked to point to where the pain began and where it moved. The patient usually points from periumbillical region to right iliac fossa.

\section{Abdominal and Pelvic Ultrasonography}

A blind-ended, non compressible, aperistaltic tube, arising from the tip of the caecum with a gut signature and an outer transverse diameter of greater than $6 \mathrm{~mm}$ characterizes an inflamed appendix. $^{76 \text {, }}$
2. Alvarado Score

\begin{tabular}{|l|c|c|}
\hline \multicolumn{3}{|c|}{ Alvarado Scale for the Diagnosis of Appendicitis } \\
\hline & Manifestations & Value \\
\hline Symptoms & $\mathbf{M}=$ Migration of pain to the RLQ & 1 \\
\hline & $\mathbf{A}=$ Anorexia & 1 \\
\hline Signs & $\mathbf{N}=$ Nausea or vomiting & 1 \\
\hline & $\mathbf{R}=$ Tenderness in RLQ & 2 \\
\hline $\begin{array}{l}\text { Laboratory } \\
\text { values }\end{array}$ & $\begin{array}{c}\mathbf{E}=\text { Elevated tenderness } \\
\left(\geq 37.3^{\circ} \mathrm{C}\right)\end{array}$ & 1 \\
\hline & $\begin{array}{c}\mathbf{L}=\text { Leukocytosis }(\mathrm{TLC} \\
\left.\geq 10000 / \mathrm{mm}^{3}\right)\end{array}$ & 2 \\
\hline & $\begin{array}{c}\mathbf{S}=\text { Shift of WBC to the left } \\
(\text { Neutrophils } \geq 75 \%)\end{array}$ & 1 \\
\hline
\end{tabular}

Patients with scores of 9 to 10 are almost certain to have appendicitis; there is little advantage in further workup, and they should go to the operating room. Patients with scores of 7 to 8 have a high likelihood of appendicitis, while scores of 5 to 6 are compatible with, but not diagnostic of appendicitis. CT scanning is certainly appropriate for patients with Alvarado scores of 5 and 6 . scores of 0 to 4 make it extremely unlikely (but not impossible) that they have appendicitis. ${ }^{16}$

\section{Treatment}

- Non operative Management:. Based on the high rate of failure with antibiotics alone, nonoperative management of acute appendicitis cannot be recommended

- Open versus Laparoscopic Appendectomy: Based on the data available, one cannot convincingly recommend either OA or LA over the other.

- Postoperative Care: Postoperative care for both the laparoscopic and open approaches is similar. Patients can be discharged when they tolerate a regular diet and oral analgesics.

\section{Materials and Methods}

1) Study Area: Department of General Surge, IGIMS, Patna,

2) Study Population: All patients admitted in General Surgery Ward of, IGIMS, PATNA with provisional clinical diagnosis of acute appendicitis. Chronic appendicitis and pediatrics populations were excluded from this study. 
3) Study Period: February 2016 - July 2017.

4) Sample Size: Total 140 patients were included in this study.

5) Study Design: It was a prospective observational study.

6) Parameters Studied:

(a) Various modes of presentation of acute appendicitis:

Pain-Site, migration, character; Anorexia, Nausea, Vomiting, Fever Constipation, Diarrhoea, Dysurea (b) D clinical signs: Tachycardia, Pyrexia, Localized tenderness in right iliac fossa (RIF), Muscle guarding over RIF, Rebound tenderness over RIF , Generalized abdominal tenderness,

Pointing sign,

Dunphy's sign

- Rovsing's sign

- Psoas sign

- Obturator sign

- Tenderness on digital rectal examination (DRE)

(c) Role of various investigative procedures in acute appendicitis:

- Total Leucocyte Count (TLC)

- As per Alvarado score, TLC $\geq 10,000 / \mathrm{mm}^{3}$ was taken as a positive cut off value.

Differential Leucocyte Count (DLC)

- As per Alvarado score, Neutrophils $\geq 75 \%$ was taken as a positive cut off value.

- C-Reactive Protein (CRP)

- A CRP value $\geq 6$ was considered positive.

- Ultrasonography (USG) of abdomen \& pelvis

- Using a linear array transducer (7-14 MHz) in Xario (Toshiba made) machine and "graded compression" technique over the right iliac fossa and the site of maximum tenderness, the landmarks of the caecum, iliac vessels and psoas muscle were identified.

(d) Role of Alvarado scoring system in diagnosis of acute appendicitis:

- Alvarado Score (Mantrels)

1. Migratory RIF pain $=1$

2. Anorexia $=1$

3. Nausea or vomiting $=1$

4. Tenderness in RIF $=2$
5. Rebound tenderness $=1$

6. Elevated temperature $\left(\geq 37.3^{\circ} \mathrm{C}\right)=1$

7. Leukocytosis (TLC $\left.\geq 10,000 / \mathrm{mm}^{3}\right)=2$

8. Shift of WBC to the left (Neutrophils $\geq 75 \%)=1$

Total value $=10$

Positive cut off value was taken as $\geq 7$

\section{7) Analysis of Data}

Results of the study are compiled, tabulated and compared with the known data, statistical analysis done and inference drawn.

\section{Results and Analysis}

A total of 140 patients with clinical impression of acute appendicitis were included in this study.

Out of total 140 patients with provisional clinical diagnosis of acute appendicitis, $\mathbf{1 3 2}$ patients were subjected to emergency appendectomy. In the rest 8 patients emergency appendectomy was not done, the reasons were: 2 patients had palpable appendicular lump on clinical examination, 1 patient had right ureteric calculus on X-ray, 2 patients had alternate diagnosis on USG (1 case of ruptured ectopic pregnancy, 1 case of right sided twisted ovarian cyst) and in the remaining 2 patients, where USG was not done, the diagnosis of acute appendicitis changed after laparotomy because in one case ruptured ectopic pregnancy was found and in another case right sided twisted ovarian cyst was detected.

The removed appendices were sent for histopathological examination in all 132 cases, out of which, 103 patients were found to have histologically proven acute appendicitis. The rest 29 patients did not have any significant features of acute inflammation histologically, thus being labeled as normal appendices.

Of these 29 patients found to have normal appendices histologically, only 7 had some other detectable disease at the time of operation, namely, 3 cases of acute mesenteric lymphadenitis, 1 case of meckel's diverticulitis, 1 case of Crohn's disease, 1 case of right sided salpingitis and 1 case of caecal diverticulitis. The rest 22 patients had no detectable disease at all. 
- Clinical Diagnostic Accuracy (CDA)

Appendicitis group

$=$ x 100

Total no. of cases with clinical diagnosis of acute appendicitis

$=(105 / 140) \times 100=\mathbf{7 5 . 0 0 \%}$

- Negative Appendectomy Rate (NAR)

Total no. of histologically normal appendices

Total appendectomy done
$=\quad(29 / 134) \times 100=\mathbf{2 1 . 6 4 \%}$

- Symptoms

Table VI Symptoms $(\mathrm{n}=140)$

\begin{tabular}{|l|c|c|c|c|c|c|}
\hline \multirow{2}{*}{ Symptoms } & \multicolumn{2}{|c|}{$\begin{array}{c}\text { Appendicitis group } \\
(\mathbf{n = 1 0 5})\end{array}$} & \multicolumn{2}{c|}{$\begin{array}{c}\text { Non-appendicitis } \\
\text { group (n=35) }\end{array}$} & \multicolumn{2}{c|}{$\begin{array}{c}\text { Total } \\
(\mathbf{n = 1 4 0})\end{array}$} \\
\cline { 2 - 7 } & $\mathbf{N o .}$ & $\mathbf{\%}$ & No. & $\mathbf{\%}$ & No. & \% \\
\hline \multirow{2}{*}{$\begin{array}{l}\text { RIF pain } \\
\text { Migratory }\end{array}$} & $\mathbf{1 0 1}$ & 96.19 & 33 & 94.29 & 134 & 95.71 \\
\cline { 2 - 7 } & 66 & 62.86 & 13 & 37.14 & 79 & 56.43 \\
\cline { 2 - 7 } & 35 & 33.33 & 20 & 57.14 & 55 & 39.29 \\
\hline Gen-migratory & 14 & 13.33 & 5 & 14.29 & 19 & 13.57 \\
\hline Right sided flank pain & 24 & 22.86 & 8 & 22.86 & 32 & 22.86 \\
\hline Suprapubic pain & 16 & 15.24 & 8 & 22.86 & 24 & 17.14 \\
\hline Anorexia & $\mathbf{8 2}$ & 78.10 & 27 & 77.14 & 109 & 77.86 \\
\hline Nausea & $\mathbf{7 0}$ & 66.67 & 26 & 74.29 & 96 & 68.57 \\
\hline Vomiting & 49 & 46.67 & 11 & 31.43 & 60 & 42.86 \\
\hline Fever & 69 & 65.71 & 25 & 71.43 & 94 & 67.14 \\
\hline Constipation & 30 & 28.57 & 7 & 20.00 & 37 & 26.43 \\
\hline Diarrhoea & 15 & 14.29 & 6 & 17.14 & 21 & 15.00 \\
\hline Dysurea & 20 & 19.05 & 7 & 20.00 & 27 & 19.29 \\
\hline
\end{tabular}

\section{Physical Signs}

Table VII Physical Signs ( $n=140)$

\begin{tabular}{|l|c|c|c|c|c|c|}
\hline \multirow{2}{*}{ Physical signs } & \multicolumn{2}{|c|}{$\begin{array}{c}\text { Appendicitis group } \\
(\mathbf{n = 1 0 5})\end{array}$} & $\begin{array}{c}\text { Non-appendicitis group } \\
(\mathbf{n = 3 5})\end{array}$ & \multicolumn{2}{c|}{$\begin{array}{c}\text { Total } \\
(\mathbf{n = 1 4 0})\end{array}$} \\
\cline { 2 - 7 } & $\mathbf{N o .}$ & $\mathbf{\%}$ & $\mathbf{N o .}$ & $\mathbf{\%}$ & No. & \% \\
\hline Tachycardia (Heart rate $>100 / \mathrm{min})$ & 64 & 60.95 & 17 & 48.57 & 81 & 57.86 \\
\hline Pyrexia (Temperature $\left.\geq 37.3^{\mathbf{0}} \mathrm{C}\right)$ & 69 & 65.71 & 25 & 71.43 & 94 & 67.14 \\
\hline Tenderness in RIF & $\mathbf{1 0 1}$ & 96.19 & 32 & 91.43 & 133 & 95.00 \\
\hline Muscle guarding over RIF & 53 & 50.48 & 12 & 34.29 & 65 & 46.43 \\
\hline Rebound tenderness over RIF & 60 & 57.14 & 16 & 45.71 & 76 & 54.29 \\
\hline Generalized abdominal tenderness & 5 & 4.76 & 3 & 8.57 & 8 & 5.71 \\
\hline Pointing sign & $\mathbf{6 6}$ & 62.86 & 13 & 37.14 & 79 & 56.43 \\
\hline Dunphy's sign & 49 & 46.67 & 19 & 54.29 & 68 & 48.57 \\
\hline Rovsing's sign & 51 & 48.57 & 12 & 34.29 & 63 & 45.00 \\
\hline Psoas sign & 39 & 37.14 & 6 & 17.14 & 45 & 32.14 \\
\hline Obturator sign & 22 & 20.95 & 2 & 5.71 & 24 & 17.14 \\
\hline Tenderness on DRE & 11 & 10.48 & 5 & 14.29 & 16 & 11.43 \\
\hline
\end{tabular}


- Total Leukocyte Count (TLC)

Table VIII Preoperative TLC Count $(n=134)$

\begin{tabular}{|l|c|c|c|c|c|c|}
\hline \multirow{2}{*}{ TLC $/ \mathbf{m m}^{\mathbf{3}}$} & \multicolumn{2}{|c|}{$\begin{array}{c}\text { Appendicitis group } \\
(\mathbf{n = 1 0 5})\end{array}$} & $\begin{array}{c}\text { Non-appendicitis group } \\
(\mathbf{n = 3 5})\end{array}$ & \multicolumn{2}{c|}{$\begin{array}{c}\text { Total } \\
\text { (n=140) }\end{array}$} \\
\cline { 2 - 7 } & No. & $\mathbf{\%}$ & No. & \% & No. & \% \\
\hline$<7,000$ & 5 & 4.76 & 10 & 28.57 & 15 & 10.71 \\
\hline 7,000 to $<10,000$ & 11 & 10.48 & 18 & 51.43 & 29 & 20.71 \\
\hline 10,000 to $<13,000$ & 34 & 32.38 & 5 & 14.29 & 39 & 27.86 \\
\hline 13,000 to $<16,000$ & 32 & 30.48 & 2 & 5.71 & 34 & 24.29 \\
\hline$\geq 16,000$ & 23 & 21.90 & 0 & 0.00 & 23 & 16.43 \\
\hline
\end{tabular}

According to Alvarado score, the positive cut off value of TLC is a count of $\geq 10,000 / \mathrm{mm}^{3}$. Taking

this as a cut off value, the diagnostic accuracy of TLC became as follows:

Table IX Diagnostic Accuracy of TLC Taking Cut off value $\geq 10,000 / \mathrm{mm}^{3}(\mathrm{n}=140)$

\begin{tabular}{|l|c|c|c|}
\hline TLC $/ \mathbf{m m}^{\mathbf{3}}$ & Appendicitis group & Non-appendicitis group & Total \\
\hline$\geq 10,000$ & $89(\mathrm{TP})$ & $7(\mathrm{FP})$ & 96 \\
\hline$<10,000$ & $16(\mathrm{FN})$ & $28(\mathrm{TN})$ & 44 \\
\hline Total & 105 & 35 & 140 \\
\hline
\end{tabular}

Sensitivity $=(\mathrm{TP} / \mathrm{TP}+\mathrm{FN}) \times 100=(89 / 105) \mathrm{x}$ $100=84.76 \%$

* Specificity $=(\mathrm{TN} / \mathrm{FP}+\mathrm{TN}) \times 100=(28 / 35) \times$ $100=80.00 \%$

- Positive predictive value $=(\mathrm{TP} / \mathrm{TP}+\mathrm{FP}) \times 100$ $=(89 / 96) \times 100=92.71 \%$
* Negative predictive value $=(\mathrm{TN} / \mathrm{FN}+\mathrm{TN}) \mathrm{x}$ $100=(28 / 44) \times 100=63.64 \%$

* $\%$ of false negative $=(\mathrm{FN} / \mathrm{TP}+\mathrm{FN}) \times 100=$ $(16 / 105) \times 100=15.24 \%$

* $\%$ of false positive $=(\mathrm{FP} / \mathrm{FP}+\mathrm{TN}) \times 100=$ $(7 / 35) \times 100=20.0$

\section{Differential Leukocyte Count (DLC)}

Table X Preoperative Neutrophils Count in \% $(n=140)$

\begin{tabular}{|l|c|c|c|c|c|c|}
\hline \multirow{2}{*}{$\begin{array}{l}\text { Neutrophils count in } \\
\%\end{array}$} & \multicolumn{2}{|c|}{$\begin{array}{c}\text { Appendicitis group } \\
(\mathbf{n = 1 0 5})\end{array}$} & \multicolumn{2}{c|}{$\begin{array}{c}\text { Non-appendicitis } \\
\text { group (n=35) }\end{array}$} & \multicolumn{2}{c|}{$\begin{array}{c}\text { Total } \\
(\mathbf{n = 1 4 0})\end{array}$} \\
\cline { 2 - 7 } & No. & $\%$ & No. & \% & No. & \% \\
\hline $65 \%-69 \%$ & 9 & 8.57 & 18 & 51.43 & 27 & 19.29 \\
\hline $70 \%-74 \%$ & 12 & 11.43 & 13 & 37.14 & 25 & 17.86 \\
\hline $75 \%-79 \%$ & 43 & 40.95 & 4 & 11.43 & 47 & 33.57 \\
\hline $80 \%-84 \%$ & 34 & 32.38 & 0 & 0.00 & 34 & 24.29 \\
\hline$\geq 85 \%$ & 7 & 6.67 & 0 & 0.00 & 7 & 5.00 \\
\hline
\end{tabular}

In our study, majority of the patients of acute appendicitis group had a neutrophils count between $75 \%$ - $79 \%$ (40.95\%), followed by between $80 \%-84 \%(32.38 \%)$. In the nonappendicitis group, on the contrary, majority had a neutrophils count between 65\% - 69\% (51.43\%), followed by between $70 \%-74 \%(37.14 \%)$.

According to Alvarado score, the positive cut off value is $\geq 75 \%$. Taking this as a cut off value, the diagnostic accuracy of neutrophils became as follows:

Table XI Diagnostic Accuracy of Neutrophils Count Taking Cut off value $\geq \mathbf{7 5 \%}$ ( $\mathbf{n = 1 3 4}$ )

\begin{tabular}{|l|c|c|c|}
\hline Neutrophils count in \% & Appendicitis group & Non-appendicitis group & Total \\
\hline$\geq 75 \%$ & $84(\mathrm{TP})$ & $4(\mathrm{FP})$ & 88 \\
\hline$<75 \%$ & $21(\mathrm{FN})$ & $31(\mathrm{TN})$ & 52 \\
\hline Total & 105 & 35 & 140 \\
\hline
\end{tabular}

(TP = True positive, $\mathrm{FP}=$ False positive, $\mathrm{TN}=$ True negative, $\mathrm{FN}=$ False negative $)$

Sensitivity $=(\mathrm{TP} / \mathrm{TP}+\mathrm{FN}) \times 100=(84 / 105) \mathrm{x}$

$100=80.00 \%$
* Specificity $=(\mathrm{TN} / \mathrm{FP}+\mathrm{TN}) \times 100=(31 / 35) \times$ $100=88.57 \%$ 
Positive predictive value $=(\mathrm{TP} / \mathrm{TP}+\mathrm{FP}) \times 100$ $=(84 / 88) \times 100=95.45 \%$

* Negative predictive value $=(\mathrm{TN} / \mathrm{FN}+\mathrm{TN}) \mathrm{x}$ $100=(31 / 52) \times 100=59.62 \%$
* $\%$ of false negative $=(\mathrm{FN} / \mathrm{TP}+\mathrm{FN}) \times 100=$ $(21 / 105) \times 100=20.00 \%$

* \% of false positive $=(\mathrm{FP} / \mathrm{FP}+\mathrm{TN}) \times 100=$ $(4 / 35) \times 100=11$.

\section{C-Reactive Protein (CRP)}

Table XII Preoperative CRP Value in $\mathrm{mg} / \mathrm{L}(\mathrm{n}=140)$

\begin{tabular}{|l|c|c|c|c|c|c|}
\hline \multirow{2}{*}{ CRP value in mg/L } & \multicolumn{2}{|c|}{$\begin{array}{c}\text { Appendicitis group } \\
(\mathbf{n = 1 0 5})\end{array}$} & \multicolumn{2}{c|}{$\begin{array}{c}\text { Non-appendicitis group } \\
(\mathbf{n = 3 5})\end{array}$} & \multicolumn{2}{c|}{$\begin{array}{c}\text { Total } \\
(\mathbf{n = 1 4 0})\end{array}$} \\
\cline { 2 - 7 } & No. & \% & No. & \% & No. & \% \\
\hline$<6$ & 8 & 7.62 & 30 & 85.71 & 38 & 27.14 \\
\hline $6-50$ & 76 & 72.38 & 5 & 14.29 & 81 & 57.86 \\
\hline $50-100$ & 17 & 16.19 & 0 & 0.00 & 17 & 12.14 \\
\hline$>100$ & 4 & 3.81 & 0 & 0.00 & 4 & 2.86 \\
\hline
\end{tabular}

CRP is considered as positive when the value is $\geq 6$ $\mathrm{mg} / \mathrm{L}$. In our study majority of the patients with acute appendicitis had a positive CRP value $72.38 \%$ between $6-50,16.19 \%$ between $50-100$ and $3.81 \%>100$. Values were higher in perforated appendicitis. On the other hand only $14.29 \%$ of patients of non-appendicitis group had a positive CRP value.

Table XIII Diagnostic Accuracy of CRP Taking Cut off value $\geq 6$ mg/L ( $n=140$ )

\begin{tabular}{|l|c|c|c|}
\hline CRP value in mg/L & Appendicitis group & Non-appendicitis group & Total \\
\hline$\geq 6$ & $97(\mathrm{TP})$ & $5(\mathrm{FP})$ & 102 \\
\hline$<6$ & $8(\mathrm{FN})$ & $30(\mathrm{TN})$ & 38 \\
\hline Total & 105 & 35 & 140 \\
\hline
\end{tabular}
Sensitivity $=(\mathrm{TP} / \mathrm{TP}+\mathrm{FN}) \times 100=(97 / 105) \mathrm{x}$
- Negative predictive value $=(\mathrm{TN} / \mathrm{FN}+\mathrm{TN}) \mathrm{x}$ $100=92.38 \%$
* Specificity $=(\mathrm{TN} / \mathrm{FP}+\mathrm{TN}) \times 100=(30 / 35) \times$ $100=85.71 \%$ $100=(30 / 38) \times 100=78.95 \%$
* $\%$ of false negative $=(\mathrm{FN} / \mathrm{TP}+\mathrm{FN}) \times 100=$ $(8 / 105) \times 100=7.62 \%$
* Positive predictive value $=(\mathrm{TP} / \mathrm{TP}+\mathrm{FP}) \times 100$
$=(97 / 102) \times 100=95.10 \%$
* $\%$ of false positive $=(\mathrm{FP} / \mathrm{FP}+\mathrm{TN}) \times 100=$ $(5 / 35) \times 100=14.29 \%$

\section{Alvarado Score}

Table XIV Alvarado Score $(n=140)$

\begin{tabular}{|l|c|c|c|c|c|c|}
\hline \multirow{2}{*}{ Alvarado score } & \multicolumn{2}{|c|}{$\begin{array}{c}\text { Appendicitis group } \\
(\mathbf{n = 1 0 5})\end{array}$} & \multicolumn{2}{c|}{$\begin{array}{c}\text { Non-appendicitis } \\
\text { group }(\mathbf{n = 3 5 )}\end{array}$} & \multicolumn{2}{c|}{$\begin{array}{c}\text { Total } \\
(\mathbf{n = 1 4 0 )}\end{array}$} \\
\cline { 2 - 7 } & No. & \% & No. & \% & No. & \% \\
\hline $9-10$ & 36 & 34.29 & 1 & 2.86 & 37 & 26.43 \\
\hline $7-8$ & 57 & 54.29 & 9 & 25.71 & 66 & 47.14 \\
\hline $5-6$ & 11 & 10.48 & 12 & 34.29 & 23 & 16.43 \\
\hline$\leq 4$ & 1 & 0.95 & 13 & 37.14 & 14 & 10.00 \\
\hline
\end{tabular}

In this study $54.29 \%$ of patients with acute appendicitis had Alvarado score between $7-8$ and $34.29 \%$ of patients with acute appendicitis had Alvarado score between $9-10$. On the other hand majority of the patients of non-appendicitis group had a score below 7 .
As a score of $\geq 7$ had a higher chance of appendicitis, it had been taken as a positive cut off value. Being so, the diagnostic accuracy of Alvarado score became as follows: 
Table XV Diagnostic Accuracy of Alvarado Score Taking Cut off value $\geq 7(n=140)$

\begin{tabular}{|l|c|c|c|}
\hline Alvarado score & Appendicitis group & Non-appendicitis group & Total \\
\hline$\geq 7$ & $93(\mathrm{TP})$ & $10(\mathrm{FP})$ & 103 \\
\hline$<7$ & $12(\mathrm{FN})$ & $25(\mathrm{TN})$ & 37 \\
\hline Total & 105 & 35 & 140 \\
\hline
\end{tabular}

( TP $=$ True positive, $\mathrm{FP}=$ False positive, $\mathrm{TN}=$ True negative, $\mathrm{FN}=$ False negative $)$

Sensitivity $=(\mathrm{TP} / \mathrm{TP}+\mathrm{FN}) \times 100=(93 / 105) \times$ $100=88.57 \%$

- Specificity $=(\mathrm{TN} / \mathrm{FP}+\mathrm{TN}) \times 100=(25 / 35) \times$ $100=71.43 \%$

* Positive predictive value $=(\mathrm{TP} / \mathrm{TP}+\mathrm{FP}) \times 100$ $=(93 / 103) \times 100=90.29 \%$
* Negative predictive value $=(\mathrm{TN} / \mathrm{FN}+\mathrm{TN}) \mathrm{x}$ $100=(25 / 37) \times 100=67.57 \%$

* $\%$ of false negative $=(\mathrm{FN} / \mathrm{TP}+\mathrm{FN}) \times 100=$ $(12 / 105) \times 100=11.43 \%$

* \% of false positive $=(\mathrm{FP} / \mathrm{FP}+\mathrm{TN}) \times 100=$ $(10 / 35) \times 100=28.57 \%$

Diagnostic accuracy of USG was as follows

Table XVII Diagnostic Accuracy of USG ( $n=96$ )

\begin{tabular}{|l|c|c|c|}
\hline USG result & Appendicitis group & Non-appendicitis group & Total \\
\hline$(+) \mathrm{ve}$ & $67(\mathrm{TP})$ & $2(\mathrm{FP})$ & 69 \\
\hline$(-) \mathrm{ve}$ & $5(\mathrm{FN})$ & $22(\mathrm{TN})$ & 27 \\
\hline Total & 72 & 24 & 96 \\
\hline
\end{tabular}

$(\mathrm{TP}=$ True positive, $\mathrm{FP}=$ False positive, $\mathrm{TN}=$ True negative, $\mathrm{FN}=$ False negative $)$

- Sensitivity $=(\mathrm{TP} / \mathrm{TP}+\mathrm{FN}) \times 100=(67 / 72) \times$ $100=93.10 \%$

* Specificity $=(\mathrm{TN} / \mathrm{FP}+\mathrm{TN}) \times 100=(22 / 24) \times$ $100=91.67 \%$

* Positive predictive value $=(\mathrm{TP} / \mathrm{TP}+\mathrm{FP}) \times 100$ $=(67 / 69) \times 100=97.10 \%$

* Negative predictive value $=(\mathrm{TN} / \mathrm{FN}+\mathrm{TN}) \mathrm{x}$ $100=(22 / 27) \times 100=81.45 \%$

* $\%$ of false negative $=(\mathrm{FN} / \mathrm{TP}+\mathrm{FN}) \times 100=$ $(5 / 72) \times 100=6.94 \%$

* $\%$ of false positive $=(\mathrm{FP} / \mathrm{FP}+\mathrm{TN}) \times 100=$ $(2 / 24) \times 100=8.33 \%$

\section{- Comparison between Various Diagnostic Methods}

On comparing the accuracy of various diagnostic methods, our findings were as follows:

* Sensitivity: USG > CRP > Alvarado score > TLC > Neutrophilia

* Specificity: USG > Neutrophilia > CRP > TLC $>$ Alvarado score

* Positive predictive value: USG > Neutrophilia $>$ CRP > TLC > Alvarado score

* Negative predictive value: USG > CRP > Alvarado score $>$ TLC $>$ Neutrophilia

* \% of false negative: USG < CRP < Alvarado score $<$ TLC $<$ Neutrophilia
* \% of false positive: USG < Neutrophilia < CRP $<$ TLC $<$ Alvarado score

- Clinical diagnostic accuracy $(\mathrm{CDA})=75 \%$

* Negative appendectomy rate $(\mathrm{NAR})=21.97 \%$

\section{Summary and Conclusion}

A total of 140 patients with clinical impression of acute appendicitis were included in this study. All patients underwent thorough clinical evaluation, preoperative total leukocyte count (TLC), differential leukocyte count (DLC) and C-reactive protein (CRP) measurement along with Alvarado scoring. Preoperative ultrasonography (USG) was done in $96(68.57 \%)$ patients.

Out of 140 patients, 132 patients underwent emergency appendectomy, 4 patients underwent laparotomy for pelvic pathology (2 cases of ruptured ectopic pregnancy and 2 cases of twisted ovarian cyst), 2 patients responded on conservative management (1 case of right ureteric calculus and 1 case of right tubo-ovarian mass) and 2 patients underwent interval appendectomy following initial conservative management because of appendicular lump.

Out of 132 patients of emergency appendectomy, 103 patients had histologically inflamed appendix, the rest 29 patients had histologically normal appendix. 2 patients with appendicular lump, who 
underwent interval appendectomy, also had histologically confirmed appendicitis. Therefore, among 140 patients with clinical diagnosis of acute appendicitis, appendicitis group became 105 and non-appendicitis group became 35 (29 histologically normal appendix +5 pelvic pathology +1 ureteric calculus). Operative interventions were done in 138 patients (132 emergency appendectomies +4 emergency surgeries for pelvic pathology +2 interval appendectomy).

To conclude, the initial step of diagnosis of acute appendicitis is mainly clinical and the clinical diagnostic accuracy (CDA) in literature varies between $67 \%$ - 80\%. In our study clinical diagnostic accuracy (CDA) was found to be $75 \%$.

On application of Alvarado score, the diagnostic sensitivity increased to $88 \%$ in our study.

The addition of CRP and ultrasonography increased the diagnostic accuracy further to the extent of $90 \%-95 \%$.

Therefore, proper evaluation of patients presenting with acute abdomen with suspicion of acute appendicitis include thorough clinical examination, Alvarado scoring, C-reactive protein (CRP) measurement and ultrasonography (USG). These are particularly important in very young patients, elderly patients and females of reproductive age group. By doing these, diagnostic accuracy of acute appendicitis will increase, negative appendectomy rate will reduce and overall management will improve.

\section{References}

1. Schwartz's principles of surgery: Specific consideration. 9th ed. New York: McGraw-Hill; 2010. p.1073-1091.

2. Fitz RH. Perforating inflammation of the vermiform appendix; with special reference to its early diagnosis and treatment. Am J Med Sci 1886;92:321346.
3. McBurney CM. Experience with early operative interference in cases of disease of the vermiform appendix. N Y Med J 1889;50:676-684

4. Thomas CG. Classic Description of Disease. Springfield; 1932.

5. Bailey \& Love's short practice of surgery: Abdominal. 26th ed.

6. Sabiston textbook of surgery, The biological basis of modern surgical practice: Abdomen. 18th ed. Vol-2

7. Gilmore OJA, Jones D, Ynag Q. Appendicitis and mimicking conditions. Lancet 1975;II:421-4.

8. Kalan M, Talbot D, Cunliffe WJ, Rich AJ. Evaluation of the modified Alvarado score in the diagnosis of acute appendicitis: a prospective study. Ann R Coll Surg 1994;76:418-9. 